\title{
Berberine sensitizes mutliple human cancer cells to the anticancer effects of doxorubicin in vitro
}

\author{
NANNAN TONG ${ }^{1 *}$, JIE ZHANG $^{2 *}$, YOURAN CHEN $^{1}$, ZHUBO LI $^{1}$, \\ YONGHUANG LUO ${ }^{1}$, HUA ZUO ${ }^{1}$ and XIAOYAN ZHAO ${ }^{1}$ \\ ${ }^{1}$ College of Pharmaceutical Sciences, Southwest University, Chongqing 400716; \\ ${ }^{2}$ Department of Neurology, Chongqing Three Gorges Central Hospital, Chongqing 404000, P.R. China
}

Received December 6, 2011; Accepted March 5, 2012

DOI: $10.3892 / 01.2012 .644$

\begin{abstract}
The clinical use of doxorubicin (DOX), a potent antineoplastic agent, is limited by its serious side-effects, which include acute and chronic cumulative dose-related cardiotoxicity. Berberine (BER), a botanical alkaloid, has been reported to possess cardioprotective and antitumor effects. The 3-(4,5-dimethyl-2-thiazolyl)-2,5-diphenyl-2-tetrazolium bromide (MTT) assay was used to detect the cell viability of A549, HeLa and HepG2 cells after each cell line was treated with DOX, BER or a combination of DOX and BER for $24 \mathrm{~h}$. Apoptosis was evaluated by acridine orange staining. The results showed that BER and DOX exhibited dose-dependent inhibitory effects on A549 and HeLa cells which were likely mediated by inducing apoptosis. The same result was found in the combination group. Isobologram illustration and combination index $(\mathrm{CI})$ analyses revealed that the combination of DOX and BER generates synergistic effects in A549 $(\mathrm{CI}=0.61)$ and HeLa $(\mathrm{CI}=0.73)$ cells. These findings indicate that BER sensitizes cells to the anticancer effects of DOX.
\end{abstract}

\section{Introduction}

Doxorubicin (DOX), an anthracycline antibiotic and antineoplastic agent, was first isolated from Streptomyces peucetius (1). DOX is a potent chemotherapeutic agent that is used in the treatment of solid tumors and malignant hematological diseases (2). DOX exerts its antitumor activity by inserting into DNA, leading to double-stranded DNA breaks (DSB), and

Correspondence to: Dr Xiaoyan Zhao, College of Pharmaceutical Sciences, Southwest University, 2 Tiansheng Road, Chongqing 400716, P.R. China

E-mail: zxysandy67@yahoo.com.cn

${ }^{*}$ Contributed equally

Abbreviations: $\mathrm{AO}$, acridineorange; $\mathrm{BER}$, berberine; $\mathrm{CI}$,combination index; DOX, doxorubicin; DMSO, dimethyl sulfoxide; MTT, 3-(4,5-dimethyl-2-thiazolyl)-2,5-diphenyl-2-tetrazolium bromide; $\mathrm{IC}_{50}$, the $50 \%$ growth inhibition concentration

Key words: berberine, doxorubicin, synergistic effect, A549, HeLa intercepting DNA topoisomerase II activity $(3,4)$. However, the clinical use of DOX has been largely restricted due to its cardiotoxicity, which may lead to the development of cardiomyopathy and ultimately congestive heart failure (5). The molecular mechanisms underlying DOX-induced cardiotoxicity include the formation of free radicals, activation of transcription factor $\mathrm{NF}-\kappa \mathrm{B}$, increased lipid peroxidation and $\mathrm{Ca}^{2+}$ overloading (6-8). The use of cardioprotective drugs is an alternative approach to reduce the cardiotoxicity of DOX. Pharmacological and clinical attempts to reduce the cardiotoxicity of DOX have had little success thus far. Consequently, it is important to develop a therapy to reduce DOX-induced cardiotoxicity and increase the antitumor effect of DOX.

Berberine (BER), a botanical alkaloid, is purified from the roots and bark of the Berberis species (9). BER reportedly possesses multiple biological and pharmacological properties, including anti-diarrheal, anti-fungal, anti-diabetic (10-12), hepatoprotective and cardioprotective effects. The possible mechanism of the hepatoprotective effect is that BER inhibits the activity of CYP $2 \mathrm{E} 1$ and CYP 1A2, reduces the production of nitric oxide and lowers the AST and ALT levels in serum $(13,14)$. For the cardioprotective property, BER is known to modulate $\mathrm{Cdk} 9$ and cyclin $\mathrm{T} 1$ protein expression. BER possesses muscarinic agonist-like properties which may contribute to a reduction in myocardial damage (15-17). BER also suppresses tumor growth through the induction of apoptosis and cell cycle arrest in cancer cells (18-21). Notably, it has been reported that the acute toxicity of BER was not observed at normal dosage in mice (22).

Based on these findings, we hypothesized that combining DOX with BER as a novel strategy for tumor therapy would not only increase the effect of DOX, but also prevent the cardiotoxicity induced by DOX. The present study was therefore performed to test this hypothesis in A549, HepG2 and HeLa cells. Our observations revealed that BER enhances the antitumor effects of DOX in A549 and HeLa cells.

\section{Materials and methods}

Chemicals. BER was kindly provided by Professor Xue-Gang Li (Southwest University, Chongqing, China). Dimethyl sulfoxide (DMSO), trypsin, penicillin, streptomycin, 3-(4,5-dimethyl2-thiazolyl)-2,5-diphenyl-2-tetrazolium bromide (MTT) and 
acridine orange (AO) were purchased from Sigma (St. Louis, MO, USA). Fetal bovine serum was obtained from Tianhang Biotechnology Company (Zhejiang, China). DOX was purchased from Shanxi Powerdone Pharmaceutics Company (Beijing, China).

Cell culture. The human lung carcinoma A549, human cervix carcinoma HeLa and human hepatoma HepG2 cell lines were purchased from the Cell Bank of the Chinese Academy of Sciences (Shanghai, China). The cells were cultured in Dulbecco's modified Eagle's medium supplemented with $10 \%$ fetal bovine serum at $37^{\circ} \mathrm{C}$ in $5 \% \mathrm{CO}_{2}$. The cells were subcultured at $90 \%$ confluence with $0.25 \%$ trypsin (w/v) every 2-3 days.

Cell viability assay. The cells were seeded in 96-well plates at different densities: A549, 7,000 cells/well; HeLa, 6,000 cells/well; and HepG2, 8,000 cells/well. The stock solutions of DOX and BER [both dissolved in phosphate-buffered saline (PBS)] were then diluted in culture medium to obtain the desired concentrations (BER: 0, 1, 10, 100, 200, $400 \mu \mathrm{M}$; DOX: 0, 0.1, 1, 10, 100, $200 \mu \mathrm{M}$; BER+DOX: 0+0, 1+0.2, $10+2,50+10,100+20,200+60 \mu \mathrm{M})$. The MTT assay was used to detect cell viability. Briefly, $10 \mu \mathrm{l}$ of MTT (at $5 \mathrm{mg} / \mathrm{ml}$ ) was added to each well, at a final concentration of $500 \mu \mathrm{g} / \mathrm{ml}$. Following $4 \mathrm{~h}$ of incubation under standard conditions, the cell supernatants were removed. DMSO $(100 \mu \mathrm{l})$ was then added to dissolve the MTT crystals (formazan). The absorbance of the sample was read using a Bio-Rad microplate reader (model 630; Hercules, CA, USA) at $490 \mathrm{~nm}$.

Analysis of drug synergism. The combination index (CI) was calculated for the analysis of the synergistic, antagonistic or additive effects of the two drugs (23). The CI is calculated using the formula: $C I=\left[(D)_{1} /\left(D_{x}\right)_{1}\right]+\left[(D)_{2} /\left(D_{x}\right)_{2}\right]$, in which $(D)_{1}$ is the concentration of the first drug required to achieve a particular effect in the combination; $\left(D_{x}\right)_{1}$ is the concentration of the first drug that causes an identical effect alone; (D) ${ }_{2}$ is the concentration of the second drug which achieves a particular effect in the combination; $\left(D_{x}\right)_{2}$ is the concentration of the second drug that generates the same effect alone. $\mathrm{CI}>1$ indicates antagonism, $\mathrm{CI}=1$ indicates an additive effect and $\mathrm{CI}<1$ indicates synergy.

Fluorescent microscopy measurements. To detect apoptosis, A549 cells were stained with AO. The cells were seeded in 6 -well plates at a density of 800,000 cells/well. For the AO procedure, A549 cells were treated with different concentrations of BER and DOX (BER: 0, 75, 150, $300 \mu \mathrm{M}$; DOX: 0, 1.5, 3, $6 \mu \mathrm{M}$; BER+DOX: 0+0, 75+1.5, 150+3, 300+6 $\mu \mathrm{M})$ for $24 \mathrm{~h}$ and then $10 \mu \mathrm{l}$ of prepared AO working solution $(100 \mu \mathrm{g} / \mathrm{ml}$ in PBS) was added. The cells were immediately examined with a fluorescence microscope (Olympus U-RFLT50, Tokyo, Japan). Morphologically apoptotic cells were counted from 10 visual fields of 5 different areas for each group.

Statistical analysis. Values are presented as the mean \pm SEM. One-way ANOVA and the Student's t-test were performed. $\mathrm{P}<0.05$ was considered to indicate a statistically significant result.

\section{Results}

BER enhances DOX-mediated cytotoxicity in solid tumor cells. To determine the cell viability following treatment with different concentrations of DOX and BER in the three cell lines, the MTT assay was performed. The results indicate that DOX and BER significantly inhibited cell viability in A549, HeLa and HepG2 cell lines in a dose-dependent manner (Fig. 1). As shown in Fig. 1, $100 \mu \mathrm{M}$ BER caused 39.4\% inhibition in A549 cell lines and $200 \mu \mathrm{M}$ BER had an acute cytotoxic effect in A549 and HeLa cells. The 50\% growth inhibition concentration $\left(\mathrm{IC}_{50}\right)$ of BER in $\mathrm{A} 549, \mathrm{HepG} 2$ and HeLa cells following $24 \mathrm{~h}$ of incubation was 139.4, 3,587.9 and $159.5 \mu \mathrm{M}$, respectively (Table IA). The $\mathrm{IC}_{50}$ of DOX and BER in the combination group are shown in Table IB. A549 and HeLa cells were found to be more sensitive to BER than HepG2 cells (Table I). A549 cells were the most sensitive to DOX. In the present study, we found that the $\mathrm{IC}_{50}$ of the combination of BER and DOX was lower than that of each drug used alone.

Synergistic action of BER and DOX. Isobolograms were used to evaluate whether combining BER and DOX generates a synergistic effect (24). As shown in Fig. 2, the Y-axis shows the $\mathrm{IC}_{50}$ of BER and the $\mathrm{X}$-axis shows the $\mathrm{IC}_{50}$ of DOX. The straight line (additivity line) connects the $\mathrm{IC}_{50}$ values of DOX and BER when the drugs are used alone. In the present study, we found that the $\mathrm{IC}_{50}$ of combined DOX and BER was below the straight line, indicating that a combination of the two drugs may generate a synergistic antitumor effect in A549 and HeLa cells (Fig. 2). The CI was used to analyze the synergistic effect. The $\mathrm{IC}_{50}$ of DOX and BER was used to calculate CI. The CI was $(1.7 / 3.1)+(8.6 / 139.4)=0.61$ in the A549 cells, indicating that combined DOX and BER generates synergistic effect. In the HeLa cells, the CI was $(1.9 / 16.7)+(98.9 / 159.5)=0.73$. These results suggest the synergistic action of BER and DOX in cancer therapy.

Combined treatment with BER and DOX causes solid tumor cell apoptosis. To assess whether the decrease in viability was mediated by inducing apoptosis, cells that had been treated with the two drugs were stained with AO. Results showed that the single and combined treatment with DOX and BER induced apoptosis (Fig. 3). The number of apoptotic cells was increased in the combination group compared with the single treatment group (Fig. 3D), suggesting that the combination of DOX and BER synergistically induces the apoptosis of A549 cells.

\section{Discussion}

The findings of the present study indicate that BER, a botanical alkaloid, is able to enhance the anticancer effect of DOX in A549 and HeLa cells. Our results have shown that DOX and BER significantly reduced the viability of A549 and HeLa cells. From the $\mathrm{IC}_{50}$ of DOX and BER, we found that the $\mathrm{IC}_{50}$ of the combination of DOX and BER was lower than the $\mathrm{IC}_{50}$ of the drugs used singly. Notably, the results of this study demonstrate that combining DOX with BER generates a synergistic anticancer effect in A549 and HeLa cells. 
A

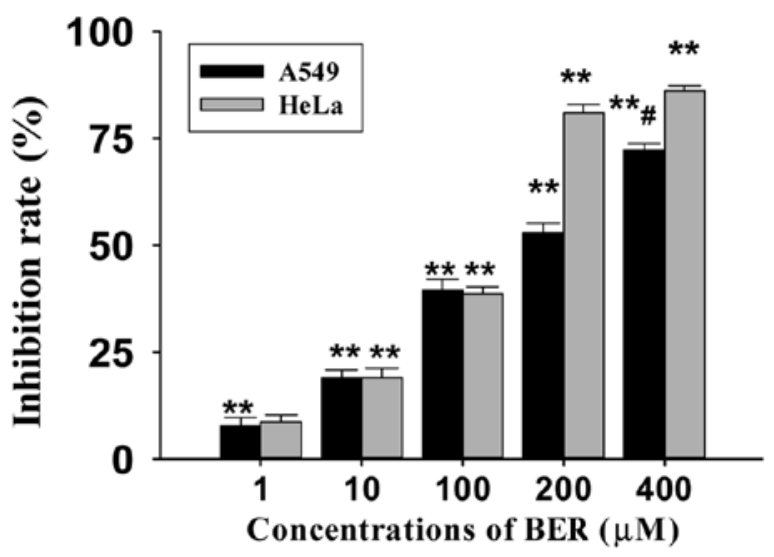

B
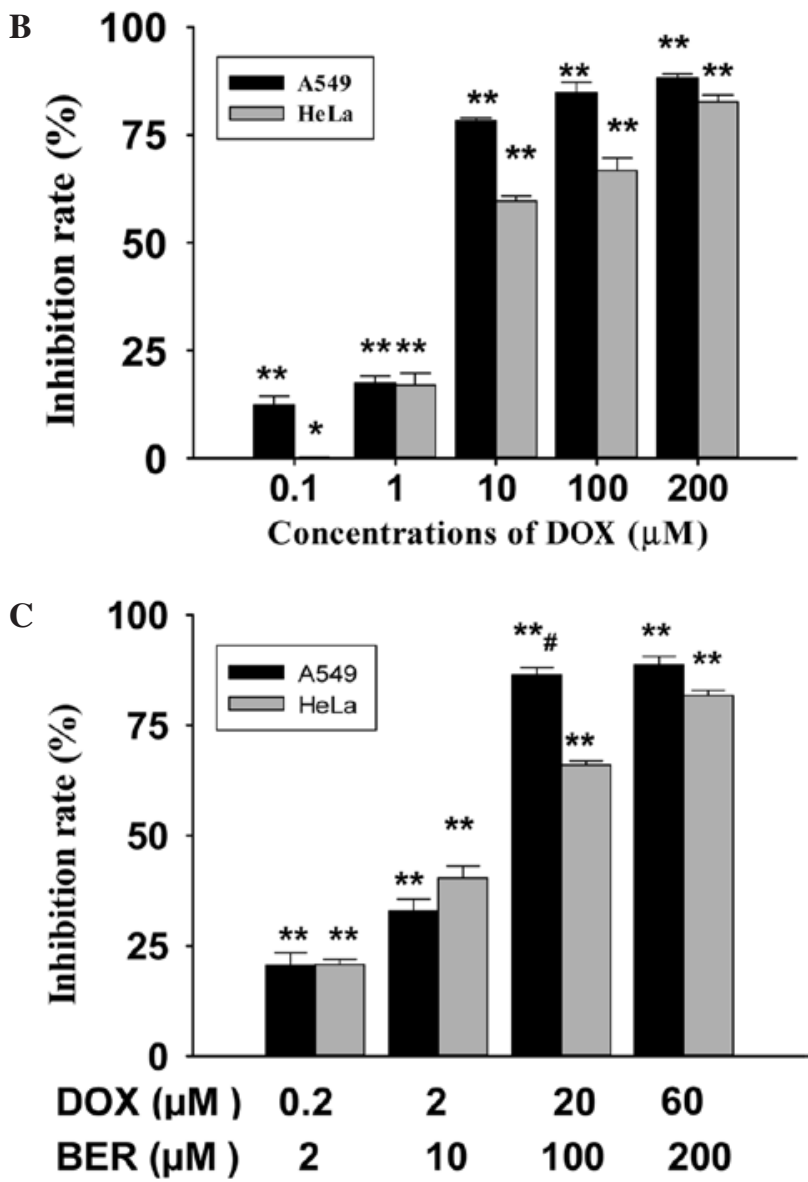

Figure 1. Inhibition rates of A549 and HeLa cells treated with (A) BER, (B) DOX and (C) a combination of DOX and BER for $24 \mathrm{~h}$. The two drugs, administered singly and in combination for $24 \mathrm{~h}$, significantly inhibited cell proliferation in A549 and HeLa cells in a dose-dependent manner. ${ }^{*} \mathrm{P}<0.05,{ }^{* *} \mathrm{P}<0.01$, compared with the control group that was treated with no drugs. (A) ${ }^{\#} \mathrm{P}<0.05$ compared with A549 cells treated with BER $(200 \mu \mathrm{M})$. (C) ${ }^{\#} \mathrm{P}<0.05$ compared with A549 cells treated with a combination of BER and DOX (200+60 $\mu \mathrm{M}$, respectively). BER berberine; DOX, doxorubicin.

DOX has been found to have anticancer activities against a range of solid tumors. However, the therapeutic use of DOX has been limited by its serious dose-related cardiotoxicity (25). BER has been reported to be safe and beneficial in the treatment of patients with chronic congestive heart failure (16). Therefore, combining DOX with BER is a novel strategy for the treatment of cancer and reduction of the cardiotoxicity induced by DOX.
Table I. Sensitivity of A549, HepG2 and HeLa cells to the treatment with BER and DOX alone and in combination.

$\mathrm{A}, \mathrm{IC}_{50}$ of BER and DOX alone $(\mu \mathrm{M})$.

\begin{tabular}{lcccc}
\hline & & A549 cells & HepG2 cells & HeLa cells \\
\hline IC $_{50}$ & BER & 139.4 & $3,587.8$ & 159.5 \\
& DOX & 3.1 & 9.2 & 16.7 \\
\hline
\end{tabular}

$\mathrm{B}, \mathrm{IC}_{50}$ of combined DOX and BER $(\mu \mathrm{M})$.

\begin{tabular}{ccccc}
\hline & & A549 cells & HepG2 cells & HeLa cells \\
\hline $\mathrm{IC}_{50}$ & BER & 8.6 & - & 98.9 \\
& DOX & 1.7 & - & 1.9
\end{tabular}

$\mathrm{IC}_{50}, 50 \%$ growth inhibition concentration; BER, berberine; DOX, doxorubicin.

A

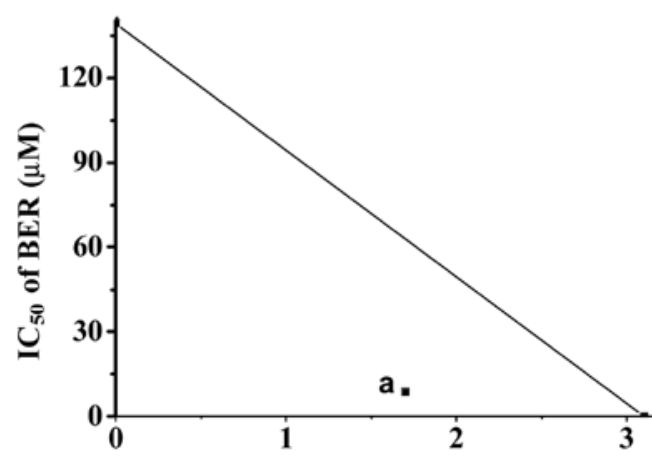

B

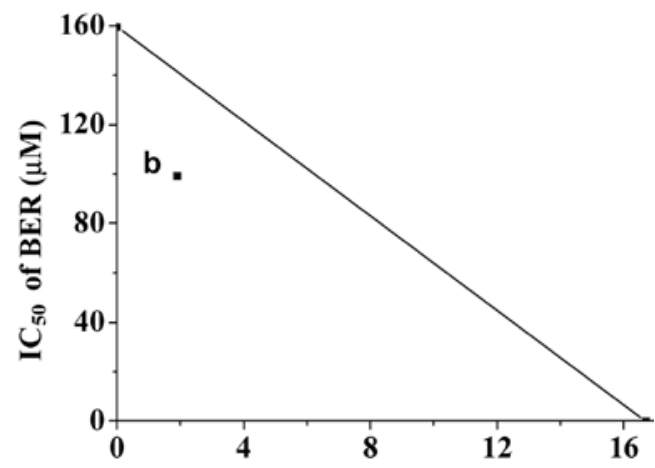

Figure 2. Isobologram for the combination of DOX with BER which generates a synergistic effect. (A) Isobologram illustration for BER+DOX-treated A549 cells; point ' $\mathrm{a}$ ' is the $\mathrm{IC}_{50}$ of combined DOX and BER. (B) Isobologram illustration for BER- and DOX-treated HeLa cells; point ' $b$ ' is the $\mathrm{IC}_{50}$ of combined DOX and BER. Both points ' $a$ ' and ' $b$ ' are below the isobologram lines. BER berberine; DOX, doxorubicin; $\mathrm{IC}_{50}, 50 \%$ growth inhibition concentration.

BER is a naturally occurring botanical alkaloid that is found in the roots and bark of the Berberis species. In clinical use, BER possesses anti-inflammatory, anti-diarrheal and anti-fungal effects. BER has also been reported to possess anticancer properties and anti-metastatic effects in non-small cell lung cancer A549 cells (26). The mechanism of its antitumor effect is that BER induces apoptosis and cell cycle arrest in cancer cells $(27,28)$. However, the anticancer effect of BER is 


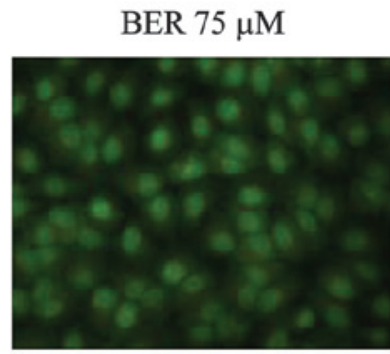

B

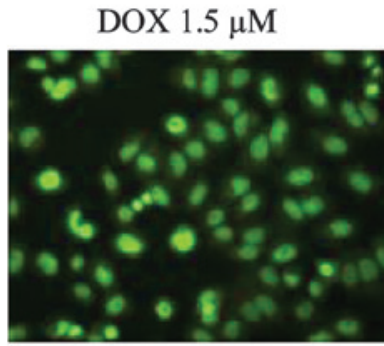

C

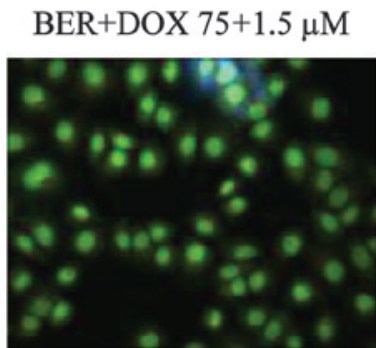

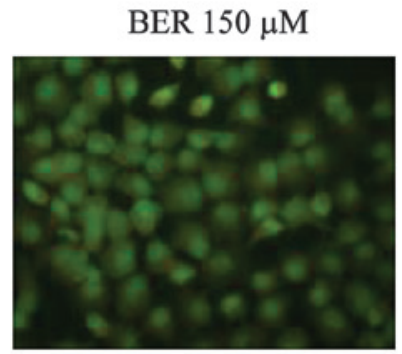

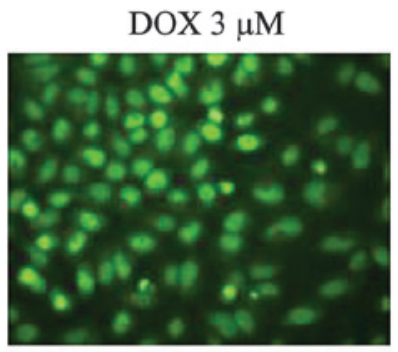

BER+DOX $150+3 \mu \mathrm{M}$

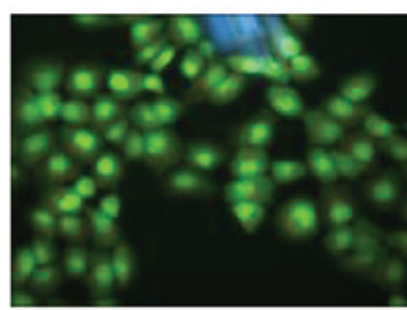

BER $300 \mu \mathrm{M}$
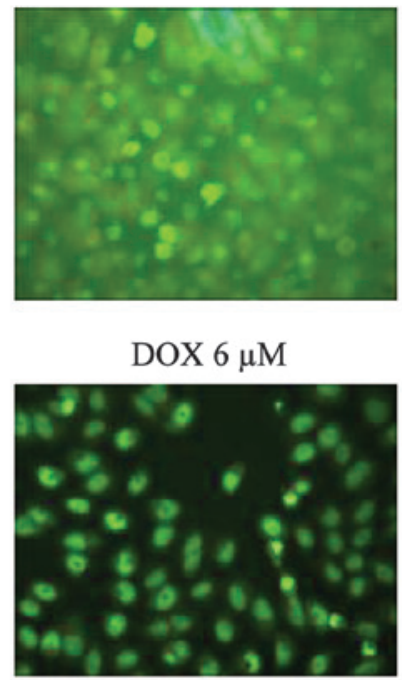

BER+DOX $300+6 \mu \mathrm{M}$

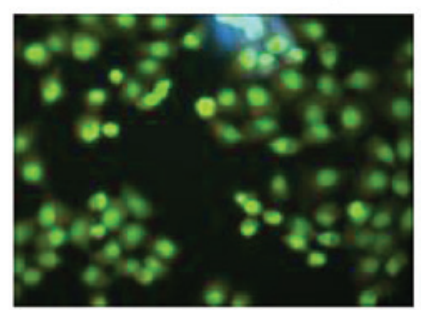

D

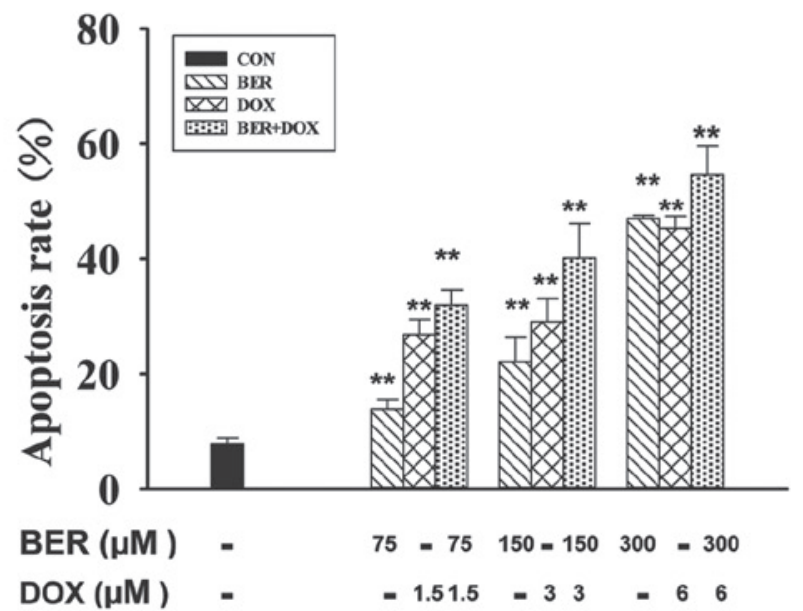

Figure 3. A549 cell apoptosis was assessed by AO staining. (A) A549 cells were preincubated with BER (75, 150, $300 \mu \mathrm{M})$ for 24 h. (B) A549 cells were preincubated with DOX $(1.5,3,6 \mu \mathrm{M})$ for $24 \mathrm{~h}$. (C) A549 cells were preincubated with DOX+BER $(1.5+75,3+150,6+300 \mu \mathrm{M})$ for $24 \mathrm{~h}$. (D) Apoptosis induced in BER-, DOX- or BER+DOX-treated cells. In the combination group, the apoptosis rates were increased compared with the single treatment groups. ${ }^{* *} \mathrm{P}<0.01$, compared with the control group that was treated with no drugs. $\mathrm{N}=10$. BER berberine; DOX, doxorubicin; AO, acridine orange.

associated with the cell type; the $\mathrm{IC}_{50}$ of BER in the HepG2 cell line is 3,587.8 $\mu \mathrm{M}$, which is extremely high for an antitumor drug, but the $\mathrm{IC}_{50}$ of BER is lower in A549 and HeLa cells. Our results have shown that BER induces apoptosis in A549 cells. Notably, the combination of DOX and BER also synergistically induced the apoptosis of A549 cells (Fig. 3). These data suggest that the induction of apoptosis is the mechanism by which the combination of DOX and BER inhibits the growth of A549 cells. However, more investigations are required to demonstrate the efficacy of the combination of DOX and BER in treating cancer patients.

The induction of apoptosis is one of the antitumor mechanisms of DOX and BER. This is in accordance with the theory of 'independent similar action' (29). Therefore, combining DOX with BER may achieve a synergistic antitumor effect. In the present study, we used isobologram illustrations to detect 
the synergism. A combination of the two drugs generated synergistic antitumor effects in A549 $(\mathrm{CI}=0.61)$ and $\mathrm{HeLa}$ (CI=0.73) cells (Fig. 3). Thus, more studies should be conducted to detect the mechanism of the synergistic anticancer action of DOX and BER.

In conclusion, we confirmed that the combination of DOX and BER synergistically generates anticancer effects in A549 and HeLa cells in vitro, possibly mediated by inducing apoptosis. With regard to HepG 2 cells, the $\mathrm{IC}_{50}$ of BER is extremely high for an antitumor agent. The combination of DOX with BER is a novel strategy that has potential in the treatment of cancer patients.

\section{Acknowledgements}

This study was supported by the National Natural Science Foundation of China (81001454), the Research Fund for the Doctoral Program of Southwest University, China (SWU109036), the Fundamental Research Funds for the Central Universities (XDJK2010C061) and the Fund for Construction of Scientific and Technical Innovation of Chongqing (CSTC, 2009CB1010).

\section{References}

1. Henderson IC and Frei TE III: Adriamycin and the heart. N Engl J Med 300: 310-312, 1979.

2. Christiansen S and Autschbach R: Doxorubicin in experimental and clinical heart failure. Eur J Cardiothorac Surg 30: 611-616, 2006.

3. Binaschi M, Capranico G, Dal Bo L and Zunino F: Relationship between lethal effects and topoisomerase II mediated doublestrand DNA breaks produced by anthracyclines with different sequence specificity. Mol Pharmacol 51: 1053-1059, 1997.

4. Hennig UG, Rudd NL and Hoar DI: Kinetochore immunofluorescence in micronuclei: a rapid method for the in situ detection of aneuploidy and chromosome breakage in human fibroblasts. Mutat Res 203: 405-414, 1988.

5. Boucek RJ Jr, Dodd DA, Atkinson JB, Oquist N and Olson RD: Contractile failure in chronic doxorubicin-induced cardiomyopathy. J Mol Cell Cardiol 29: 2631-2640, 1997.

6. Doroshow JH: Effect of anthracyline antibiotics on oxygen radical formation in rat heart. Cancer Res 43: 460-472, 1983.

7. Ichihara S, Yamada Y, Kawai Y, Osawa T, Furuhashi K, Duan Z and Ichihara G: Roles of oxidative stress and Akt signaling in doxorubicin cardiotoxicity. Biochem Biophys Res Commun 359: 27-33, 2007.

8. Temma K, Chugun A, Akera T, Hara Y, Sasaki T and Kondo H: $\mathrm{Ca}^{2+}$ overloading causes the negative inotropic effect of doxorubicin in myocytes isolated from guinea-pig hearts. Eur J Pharmacol 322: 235-242, 1997.

9. Timothy CB and Gregory SK: Berberine: therapeutic potential of an alkaloid found in several medicinal plants. Altern Med Rev 2: 94-102, 1997.

10. Takase H, Yamamoto K, Ito K and Yumioka E: Pharmacological studies on antidiarrheal effects of berberine and geranii herb. Nihon Yakurigaku Zasshi 102: 101-112, 1993 (In Japanese).
11. Iwazaki RS, Endo EH, Ueda-Nakamura T, Nakamura CV, Garcia LB and Filho BP: In vitro antifungal activity of the berberine and its synergism with fluconazole. Antonie Van Leeuwenhoek 97: 201-205, 2010.

12. Zhang H, Wei J, Xue R, et al: Berberine lowers blood glucose in type 2 diabetes mellitus patients through increasing insulin receptor expression. Metabolism 59: 285-292, 2010.

13. Zhao X, Zhang JJ, Wang X, Bu XY, Lou YQ and Zhang GL: Effect of berberine on hepatocyte proliferation, inducible nitric oxide synthase expression, cytochrome P450 2E1 and 1A2 activities in diethylnitrosamine- and phenobarbital- treated rats. Biomed Pharmacother 62: 567-572, 2008.

14. Janbaz KH and Gilani AH: Studies on preventive and curative effects of berberine on chemical-induced hepatotoxicity in rodents. Fitoterapia 71: 25-33, 2000.

15. Zhou JY, Zhou SW, Tang JL, Xu Y and Ying Y: Effect of berberine on $\mathrm{Cdk} 9$ and cyclin T1 expressions in myocardium of diabetic rats. J Med Coll PLA 23: 45-51, 2008.

16. Zeng XH, Zeng XJ and Li YY: Efficacy and safety of berberine for congestive heart failure secondary to ischemic or idiopathic dilated cardiomyopathy. Am J Cardiol 92: 173-176, 2003.

17. Salehi S and Filtz TM: Berberine possesses muscarinic agonistlike properties in cultured rodent cardiomyocytes. Pharmacol Res 63: 335-340, 2011.

18. Patil JB, Kim J and Jayaprakasha GK: Berberine induces apoptosis in breast cancer cells (MCF-7) through mitochondrialdependent pathway. Eur J Pharmacol 645: 70-78, 2010.

19. Kuo CL, Chou CC and Yung BY: Berberine complexes with DNA in the berberine-induced apoptosis in human leukemic HL-60 cells. Cancer Lett 93: 193-200, 1995.

20. Tsang CM, Lau EP, Di K, et al: Berberine inhibits Rho GTPases and cell migration at low doses but induces G2 arrest and apoptosis at high doses in human cancer cells. Int J Mol Med 24: 131-138, 2009.

21. Kim JB, Yu JH, Ko E, et al: The alkaloid berberine inhibits the growth of Anoikis-resistant MCF-7 and MDA-MB-231 breast cancer cell lines by inducing cell cycle arrest. Phytomedicine 17: 436-440, 2010.

22. Kheir MM, Wang YG, Hua L, Hu J, Li LL, Lei F and Du L: Acute toxicity of berberine and its correlation with the blood concentration in mice. Food Chem Toxicol 48: 1105-1110, 2010.

23. Chou TC and Talalay P: Quantitative analysis of dose-effect relationships: the combined effects of multiple drugs or enzyme inhibitors. Adv Enzyme Regul 22: 27-55, 1984.

24. Tallarida RJ: Drug synergism: Its detection and applications. J Pharmacol Exp Ther 298: 865-872, 2001.

25. Yoshida M, Shiojima I, Ikeda $\mathrm{H}$ and Komuro 1: Chronic doxorubicin cardiotoxicity is mediated by oxidative DNA damage-ATM-p53-apoptosis pathway and attenuated by pitavastatin through the inhibition of Racl activity. J Mol Cell Cardiol 47: 698-705, 2009.

26. Peng PL, Hsieh YS, Wang CJ, Hsu JL and Chou FP: Inhibitory effect of berberine on the invasion of human lung cancer cells via decreased productions of urokinase-plasminogen activator and matrix metalloproteinase-2. Toxicol Appl Pharmacol 214: 8-15, 2006.

27. Yan K, Zhang C, Feng J, et al: Induction of G1 cell cycle arrest and apoptosis by berberine in bladder cancer cells. Eur J Pharmacol 661: 1-7, 2011.

28. Meeran SM, Katiyar S and Katiyar SK: Berberine-induced apoptosis in human prostate cancer cells is initiated by reactive oxygen species generation. Toxicol Appl Pharmacol 229: 33-43, 2008.

29. Bliss CI: The toxicity of poisons applied jointly. Ann Appl Biol 26: 585-615, 1939. 